\title{
Entangled-photon Fourier optics
}

\author{
Ayman F. Abouraddy, Bahaa E. A. Saleh, Alexander V. Sergienko, and Malvin C. Teich \\ Quantum Imaging Laboratory, Departments of Electrical \& Computer Engineering and Physics, Boston University, \\ Boston, Massachusetts 02215-2421
}

Received June 11, 2001; revised manuscript received October 9, 2001

Entangled photons, generated by spontaneous parametric downconversion from a second-order nonlinear crystal, present a rich potential for imaging and image-processing applications. Since this source is an example of a three-wave mixing process, there is more flexibility in the choices of illumination and detection wavelengths and in the placement of object(s) to be imaged. Moreover, this source is entangled, a fact that allows for imaging configurations and capabilities that cannot be achieved by use of classical sources of light. We examine a number of imaging and image-processing configurations that can be realized with this source. The formalism that we utilize facilitates the determination of the dependence of imaging resolution on the physical parameters of the optical arrangement. (C) 2002 Optical Society of America

OCIS codes: $270.0270,190.0190,110.0110,070.0070$.

\section{INTRODUCTION}

The process of optical parametric three-wave mixing in a second-order nonlinear medium ${ }^{1-10}$ involves the coherent interaction between three optical fields with, generally, different wavelengths: pump, signal, and idler. Because of the phase-matching requirements, ${ }^{6,7,10}$ the wave vectors are related, and the spatial distributions of the fields are therefore highly coupled. This process may therefore be utilized in distributed multiwavelength imaging or image processing, where objects are placed in the path of one or more of these fields of different wavelengths and the intensities or cross correlations are measured. Two examples of phenomena based on three-wave mixing, optical parametric oscillation and optical parametric amplification, have been studied extensively, and many interesting phenomena of spatial correlation, ${ }^{11}$ pattern formation, ${ }^{12,13}$ and reduced-noise image amplification ${ }^{14,15}$ have been reported.

A third example is the process of spontaneous parametric downconversion ${ }^{16}$ (SPDC), a phenomenon that exhibits quantum entanglement. ${ }^{17}$ The signal and idler waves, created when the nonlinear medium is illuminated by an intense laser beam (pump), are produced in the form of photon pairs in an entangled quantum state (biphotons). Spatial and spectral entanglement are a consequence of the multiple possibilities for satisfying conservation of energy and momentum for each photon pair. Interest in the SPDC process has spurred many studies of its spatial and spatio-temporal photon correlation properties, ${ }^{18-28}$ and some imaging applications based on the measurement of photon-pair coincidence have been proposed $^{29,30}$ and tested. ${ }^{31,32}$

In this paper we develop a general Fourier-optics theory of image formation based on the SPDC process. In Section 2 we explore new configurations for multiwavelength distributed imaging and image-processing applications. We follow an approach introduced in a previous paper, ${ }^{33}$ in which we established a duality between partial quantum entanglement and classical partial coher- ence theory. We use the formalism developed in Ref. 33 and apply it to the image-formation process in distributed multiwavelength imaging configurations made possible by the nature of this SPDC source. In Section 3 we study the imaging resolution of these configurations and the effect on it of the various physical parameters of the system. In Appendix A we provide a brief review of classical imaging theory in the framework of the optical bilinear transformation.

\section{CONFIGURATIONS FOR SPONTANEOUS- PARAMETRIC-DOWNCONVERSION BIPHOTON IMAGING AND IMAGE PROCESSING}

The principal function of an optical imaging system is to transfer the spatial distribution of some physical property of an object (transmittance, reflectance, or absorbance), by means of an optical wave, to a remote location where it is measured with a photodetector. ${ }^{9,34}$ An imageprocessing system transforms one image into another with enhanced features or obtains a new image from more than one image, such as the correlation of two images. ${ }^{35-37}$ We examine here various configurations for imaging and image processing based on biphoton beams generated by SPDC. As mentioned above, the existence of three optical beams (pump, signal, and idler) allows us to construct imaging configurations that are not achievable with other single-beam optical sources. We may place an object that is to be imaged in either of the three beams. We may alternatively place more than one object in these beams and obtain the correlation of their images.

All these configurations include two features. The first is that they are examples of distributed imaging. ${ }^{29,30,38}$ In analogy to distributed computing, where the computation resources are distributed over a network, distributed imaging allows us to reallocate the imaging components from the particular path connecting the source to the object to be imaged. The second is that they allow the possibility of multiwavelength imaging: 
The object may be illuminated with one wavelength, whereas detection takes place at another.

The use of other nonclassical sources of light in imaging has been shown to lead to noise reduction. ${ }^{14,15,39,40}$ In this paper we direct our attention to various imaging configurations. The quantum nature of the SPDC source offers the additional advantage of noise reduction, but this is immaterial to the task at hand.

In the process of SPDC, an intense laser beam (pump) illuminates a nonlinear crystal (NLC) with quadratic nonlinear susceptibility. ${ }^{7,16}$ Some of the pump photons disintegrate into pairs of photons (known traditionally as signal and idler), which conserve the energy and momentum of the parent pump photon. Consider the situation depicted in Fig. 1. The pump beam illuminates the NLC, and the signal and idler beams are measured by the single-photon detectors $\mathrm{D}_{1}$ and $\mathrm{D}_{2}$, respectively. We assume throughout a planar source and a one-dimensional geometry in the transverse plane for the sake of simplicity but without loss of generality. Optical systems, containing objects to be imaged and any optical components, may be placed in any of the three available beams.

The signal and idler photons can be emitted from the NLC in a variety of configurations. The two photons may be emitted in two different and distinct directions, in which case each photon will pass through a different (and possibly remote) optical system; this configuration is denoted noncollinear. The two photons may be emitted in the same spatial wave packet, the collinear case, but have some distinguishing characteristic, such as frequency or polarization, whereupon they effectively pass through different optical systems if the components are dispersive or polarization dependent. The two photons are detected in the same output plane in this case. Finally, the two photons may be emitted in the same spatial wave packet and have no distinguishing characteristic, the collinear degenerate case, and thus they pass through the same optical system and are detected in the same output plane.

The coincidence rate of photon pairs at the two detectors, $\mathrm{D}_{1}$ and $\mathrm{D}_{2}$ located at positions $x_{1}$ and $x_{2}$, respectively, is proportional to the fourth-order correlation function of the fields, $G^{(2)}\left(x_{1}, x_{2}\right),{ }^{33,41}$ the biphoton rate. The signal and idler beams traverse optical systems described

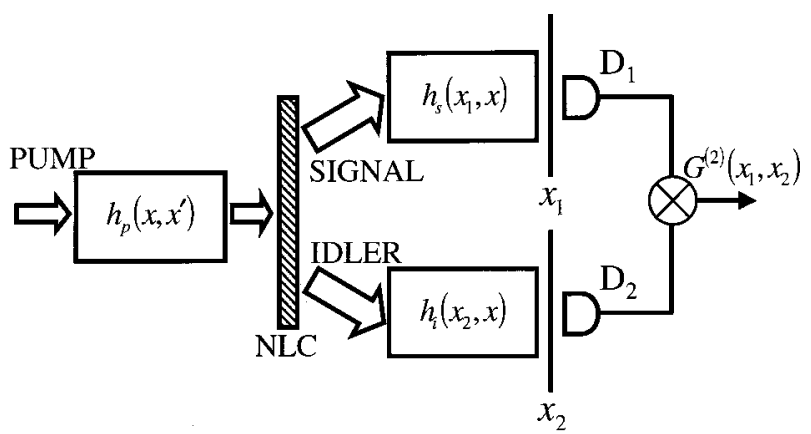

Fig. 1. Biphoton imaging using photon pairs generated by spontaneous parametric downconversion. NLC stands for nonlinear crystal; $\mathrm{D}_{1}$ and $\mathrm{D}_{2}$ are single-photon detectors at locations $x_{1}$ and $x_{2}$, respectively; $G^{(2)}\left(x_{1}, x_{2}\right)$ is the biphoton rate; $h_{p}\left(x, x^{\prime}\right)$, $h_{s}\left(x_{1}, x\right)$, and $h_{i}\left(x_{2}, x\right)$ are the impulse response functions of the optical systems placed in the paths of the pump, signal, and idler beams, respectively. by their impulse-response functions $h_{s}\left(x_{1}, x\right)$ and $h_{i}\left(x_{2}, x\right)$, respectively. It has been shown that the biphoton rate is given by ${ }^{33}$

$$
G^{(2)}\left(x_{1}, x_{2}\right)=\left|\psi\left(x_{1}, x_{2}\right)\right|^{2},
$$

where the biphoton amplitude $\psi\left(x_{1}, x_{2}\right)$ is

$$
\psi\left(x_{1}, x_{2}\right)=\int \mathrm{d} x E_{p}(x) h_{s}\left(x_{1}, x\right) h_{i}\left(x_{2}, x\right) ;
$$

here $E_{p}(x)$ is the spatial distribution of the pump field at the entrance to the NLC. The result in Eq. (2) was derived assuming a thin NLC and the presence of narrowband spectral filters in the optical system. These two assumptions simplify the analysis considerably without overshadowing the physics of the imaging processes discussed. They will be relaxed in Section 3 .

An interpretation of Eq. (2) that is useful in understanding the behavior of such a system was advocated by Klyshko $18,20,23,29$ under the name "advanced wave interpretation." In this picture, the biphoton amplitude in Eq. (2) can be viewed as the impulse-response function of an optical system represented by the cascade of three systems: propagation from $\mathrm{D}_{1}$ at $x_{1}$ back through a system with impulse-response function $h_{s}^{r}\left(x, x_{1}\right)=h_{s}\left(x_{1}, x\right)$, modulation by the pump field $E_{p}$, and subsequent transmission through a system with impulse-response function $h_{i}\left(x_{2}, x\right)$. An intuitive advantage can be gained by unfolding the system in this way, as will become clear shortly.

Two special correlation functions deriving from $G^{(2)}\left(x_{1}, x_{2}\right)$ in Eq. (1) are of interest: the marginal coincidence rate, $I^{(2)}\left(x_{2}\right)$, and the conditional coincidence rate, $I_{0}^{(2)}\left(x_{2}\right)$, defined by

$$
\begin{aligned}
& I^{(2)}\left(x_{2}\right)=\int \mathrm{d} x_{1} G^{(2)}\left(x_{1}, x_{2}\right), \\
& I_{0}^{(2)}\left(x_{2}\right)=G^{(2)}\left(0, x_{2}\right) .
\end{aligned}
$$

The marginal coincidence rate $I^{(2)}\left(x_{2}\right)$ is proportional to the probability of detecting a photon at $x_{2}$ by $\mathrm{D}_{2}$ when detector $\mathrm{D}_{1}$ detects a photon at any location $-\infty<x_{1}<\infty$. The conditional coincidence rate $I_{0}^{(2)}\left(x_{2}\right)$ is proportional to the probability of detecting a photon at $x_{2}$ by $\mathrm{D}_{2}$ when $\mathrm{D}_{1}$ detects a photon at $x_{1}=0$.

We proceed to examine the five configurations that are possible with this optical source and explore their imaging and image-processing potential.

\section{A. Object in the Signal (or Idler) Beam}

The generalized biphoton optical system described by Eq. (2) permits the object to be placed in either the signal or the idler beams such that its transmittance (or reflectance) modifies either of the impulse-response functions $h_{s}$ or $h_{i}$. Without loss of generality, we assume that the object is placed in the signal beam. However, the choice of either beam might be dictated by wavelength considerations.

Consider the situation depicted in Fig. 2. The reversesignal system $h_{s}^{r}\left(x, x_{1}\right)=h_{s}\left(x_{1}, x\right)$ is regarded as a cascade of two linear systems of impulse-response functions $h_{1}$ and $h_{2}$ with the object $t$ sandwiched in between. The biphoton amplitude can thus be written as 


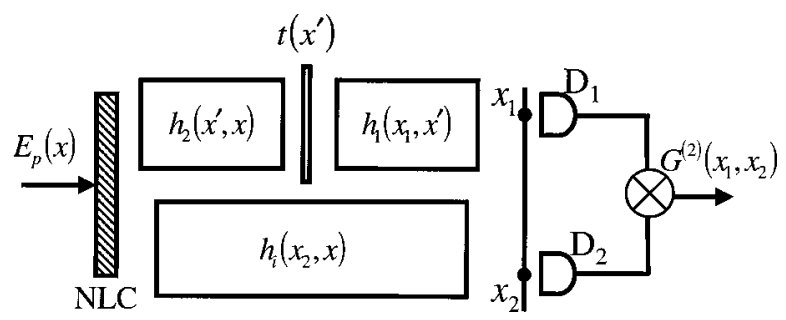

Fig. 2. Object in the signal-beam configuration. $E_{p}$ is the pump field at the entrance to the NLC; $h_{1}\left(x_{1}, x^{\prime}\right)$ and $h_{2}\left(x^{\prime}, x\right)$ are the impulse-response functions of the optical systems placed in the signal beam; $h_{i}\left(x_{2}, x\right)$ is the impulse-response function of the optical system placed in the idler beam; $t\left(x^{\prime}\right)$ is the object to be imaged, placed in the signal beam.

$$
\psi\left(x_{1}, x_{2}\right)=\int \mathrm{d} x^{\prime} t\left(x^{\prime}\right) h_{1}\left(x_{1}, x^{\prime}\right) h_{3}\left(x_{2}, x^{\prime}\right),
$$

where $h_{3}$ is the impulse-response function of a system composed of a cascade of the reverse of system $h_{2}$, an aperture $E_{p}$, and the system $h_{i}$, and is given by

$$
h_{3}\left(x_{2}, x^{\prime}\right)=\int \mathrm{d} x E_{p}(x) h_{i}\left(x_{2}, x\right) h_{2}\left(x^{\prime}, x\right) .
$$

Equation (5) states that the overall system is composed of an illumination system $h_{1}$ illuminating the object $t$, followed by an imaging system $h_{3}$, which is dependent on $h_{2}, h_{i}$, and $E_{p}$, in accordance with Eq. (6). The uniformity of the illumination system $h_{1}$ and the resolution of the system $h_{3}$ determine the quality of the overall imaging system.

In this configuration, then, the conditional coincidence rate, obtained by use of Eqs. (1), (4), and (5), is

$$
I_{0}^{(2)}\left(x_{2}\right)=\left|\int \mathrm{d} x^{\prime} t\left(x^{\prime}\right) h_{1}\left(0, x^{\prime}\right) h_{3}\left(x_{2}, x^{\prime}\right)\right|^{2} .
$$

This system is mathematically equivalent to a coherent optical system where the object is modulated by the illumination distribution $h_{1}\left(0, x^{\prime}\right)$ and transformed by a linear system of point-spread function $h_{3}\left(x_{2}, x^{\prime}\right)$, followed by a squarer, viz. the bilinear transformation of Eq. (A2).

On the other hand, by use of Eqs. (1), (3), and (5), the marginal coincidence rate, measured when $\mathrm{D}_{1}$ collects photons from all points in its plane (i.e., acts as a bucket detector), is given by

$$
\begin{aligned}
I^{(2)}\left(x_{2}\right)= & \int \mathrm{d} x_{1}\left|\int \mathrm{d} x^{\prime} t\left(x^{\prime}\right) h_{1}\left(x_{1}, x^{\prime}\right) h_{3}\left(x_{2}, x^{\prime}\right)\right|^{2} \\
= & \iint \mathrm{d} x^{\prime} \mathrm{d} x^{\prime \prime} t^{*}\left(x^{\prime}\right) t\left(x^{\prime \prime}\right) g\left(x^{\prime}, x^{\prime \prime}\right) \\
& \times h_{3}^{*}\left(x_{2}, x^{\prime}\right) h_{3}\left(x_{2}, x^{\prime \prime}\right) .
\end{aligned}
$$

The quadratic transformation of the object $t(x)$ in Eq. (8) is clearly the mathematical equivalent of the bilinear transformation in Eq. (A1), representing a partially coherent imaging system. The function $g\left(x^{\prime}, x^{\prime \prime}\right)$ is given by

$$
g\left(x^{\prime}, x^{\prime \prime}\right)=\int \mathrm{d} x_{1} h_{1}^{*}\left(x_{1}, x^{\prime}\right) h_{1}\left(x_{1}, x^{\prime \prime}\right)
$$

and plays the role of the coherence function of the field.
Two limiting forms of $g\left(x^{\prime}, x^{\prime \prime}\right)$ are of interest. The first is $g\left(x^{\prime}, x^{\prime \prime}\right)=\delta\left(x^{\prime}-x^{\prime \prime}\right)$, which leads to

$$
I^{(2)}\left(x_{2}\right)=\int \mathrm{d} x^{\prime}\left|t\left(x^{\prime}\right)\right|^{2}\left|h_{3}\left(x_{2}, x^{\prime}\right)\right|^{2},
$$

which is the equivalent of an incoherent imaging system [Eq. (A3)]. The other limit is $g\left(x^{\prime}, x^{\prime \prime}\right)=f^{*}\left(x^{\prime}\right) f\left(x^{\prime \prime}\right)$, in which case

$$
I^{(2)}\left(x_{2}\right)=\left|\int \mathrm{d} x^{\prime} t\left(x^{\prime}\right) f\left(x^{\prime}\right) h_{3}\left(x_{2}, x^{\prime}\right)\right|^{2},
$$

which is the equivalent of a coherent imaging system [Eq. (A2)]. We can achieve the first limit by using a $2-f$ (Fourier transform) system or a $4-f$ (imaging) system for $h_{1}$ followed by a bucket detector. Moving the bucket detector in the $2-f$ system away from the back focal plane or changing the area of the detector would lead to a gradual transition from coherent to incoherent imaging. This was performed experimentally in Ref. 21, where the change from coherent to incoherent imaging, achieved by changing the detector aperture size in one beam, was observed by monitoring the loss of the fringe visibility of a double-slit placed in the other beam.

We now examine a few examples based on this configuration that manifest its usefulness.

\section{Example: Fourier-Transform Imaging}

Consider the system illustrated in Fig. 3. The signal and idler systems are unfolded for sake of clarity. We assume for simplicity in this and in the following examples, except if indicated otherwise, that the pump and the NLC are of infinite transverse extent. The signal arm includes the object, $t$, and the system $h_{2}$ is nothing but freespace propagation at the signal wavelength $\lambda_{s}$ for a distance $d_{s}$. The idler system comprises a lens of focal length $f$ at a distance $d_{i}$ from the NLC and a distance $d_{2}$ from the detection plane, as shown in Fig. 3. Free-space propagation for the distance $d_{s}$ at $\lambda_{s}$ and for the distance $d_{i}$ at $\lambda_{i}$ may be substituted by free-space propagation for an equivalent distance $d_{1}$ at wavelength $\lambda_{i}$, where

$$
d_{1}=d_{i}+d_{s} \frac{\lambda_{s}}{\lambda_{i}} .
$$

If we take $d_{2}$ to be equal to focal length of the lens $f$, and also choose $d_{i}$ and $d_{s}$ such that $d_{1}=f$ according to Eq.

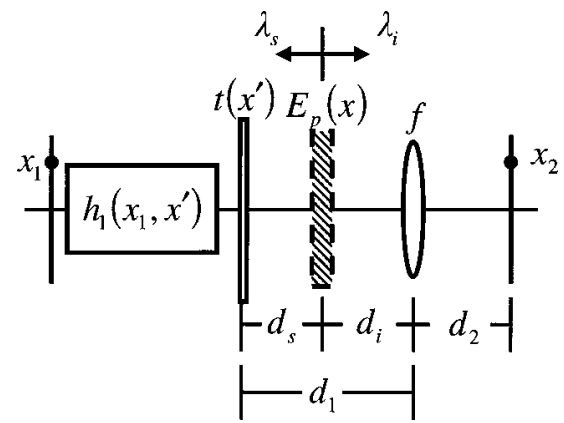

Fig. 3. Object in the signal-beam configuration of examples 1 and 2 displayed in an unfolded picture. $E_{p}, h_{1}\left(x_{1}, x^{\prime}\right)$, and $t\left(x^{\prime}\right)$ are the same as in Fig. 2; $f$ is the focal length of a lens placed in the idler beam. See text for details. 


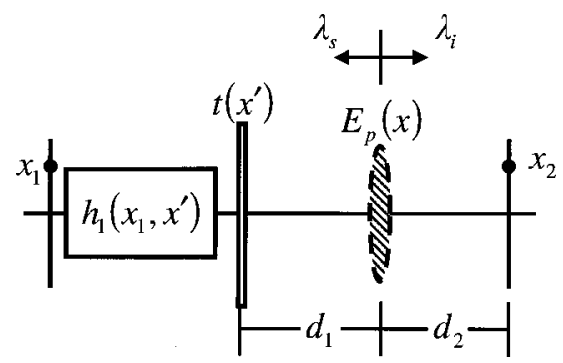

Fig. 4. Object in the signal-beam configuration of example 3 displayed in an unfolded mode. $h_{1}\left(x_{1}, x^{\prime}\right)$ and $t\left(x^{\prime}\right)$ are the same as in Fig. 2; a lens is placed in the pump beam and is represented here by the dotted lens of focal length $f$. See text for details.

(12), then the system becomes a Fourier-transform system with impulse-response function

$$
h_{3}\left(x_{2}, x^{\prime}\right)=\exp \left[j 2 \pi\left(\frac{d_{s}}{\lambda_{s}}+\frac{d_{i}+f}{\lambda_{i}}\right)\right] \exp \left(-j \frac{2 \pi}{\lambda_{i} f} x_{2} x^{\prime}\right) .
$$

If we now take the illumination system $h_{1}$ to be uniform, so that $h_{1}\left(0, x^{\prime}\right)=1$, then the overall system is a Fourier-transform system when the conditional coincidence rate is considered. Equations (7) and (13) then yield

$$
I_{0}^{2}\left(x_{2}\right)=\left|T\left(\frac{2 \pi}{\lambda_{i} f} x_{2}\right)\right|^{2}
$$

where $T(q)$ is the Fourier transform of $t(x)$. The system simply generates the diffraction pattern of the object distribution.

\section{Example: Ideal Single-Lens Imaging}

In the same configuration depicted in Fig. 3, we may choose the distance $d_{1}$, calculated according to Eq. (12), and the distance $d_{2}$ to satisfy

$$
\frac{1}{d_{1}}+\frac{1}{d_{2}}=\frac{1}{f}
$$

which is the geometrical-optics imaging equation of a thin lens of focal length $f$. In this case the impulse-response function of the system $h_{3}$ becomes

$$
\begin{aligned}
h_{3}\left(x_{2}, x^{\prime}\right)= & \exp \left[j 2 \pi\left(\frac{d_{s}}{\lambda_{s}}+\frac{d_{i}+d_{2}}{\lambda_{i}}\right)\right] \\
& \times \exp \left[j \frac{\pi x_{2}^{2}}{\lambda_{i} d_{2}}\left(1-\frac{1}{M}\right)\right] \delta\left(x_{2}-M x^{\prime}\right),
\end{aligned}
$$

where $M=-d_{2} / d_{1}$ is the magnification of the imaging system. If the illumination system $h_{1}$ is uniform, then the conditional coincidence rate $I_{0}^{(2)}\left(x_{2}\right)$ is proportional to the magnified object intensity transmittance, $\left|t\left(x_{2} / M\right)\right|^{2}$. The marginal coincidence rate $I^{(2)}\left(x_{2}\right)$, however, is $I^{(2)}\left(x_{2}\right)=g\left(x_{2} / M, x_{2} / M\right)\left|t\left(x_{2} / M\right)\right|^{2}$, where $g\left(x^{\prime}, x^{\prime \prime}\right)$ is given by Eq. (9). If $g\left(x^{\prime}, x^{\prime}\right)$ is uniform over an area larger than that of the image, $I^{(2)}\left(x_{2}\right)$ becomes proportional to the magnified object intensity transmittance as is the case for $I_{0}^{(2)}\left(x_{2}\right)$.
Note that the lens may equivalently be put in the signal beam and the distances readjusted so as to satisfy a condition similar to Eq. (15). The system developed by Pittman et al. ${ }^{31}$ is an example of this case in which the object is placed directly in the plane of $D_{1}$ so that $h_{1}\left(x_{1}, x^{\prime}\right)=\delta\left(x_{1}-x^{\prime}\right)$.

\section{Example: Lens in the Pump}

We now study another example where we manipulate the pump beam and place the object in either the signal or idler paths. An example of this configuration is the system proposed by Belinsky and Klyshko ${ }^{29}$ and demonstrated experimentally by Pittman et al. ${ }^{32}$

The configuration is shown schematically in Fig. 4. A plane-wave pump beam is focused by a lens of focal length $f$, located at a distance $d<f$ from the NLC. The pump wave front now has a radius of curvature $R=f-d$ at the NLC entrance and consequently acts as a lens or spherical mirror in the advanced wave interpretation. The signal system is composed of free-space propagation for a distance $d_{1}$ at $\lambda_{s}$, followed by the object $t$ and an optical system $h_{1}$. The idler system is simply free-space propagation for a distance $d_{2}$ at $\lambda_{i}$.

If the following relationship is satisfied by the various distances and wavelengths,

$$
\frac{1}{\lambda_{s} d_{1}}+\frac{1}{\lambda_{i} d_{2}}=\frac{1}{\lambda_{p} R}
$$

then $h_{3}\left(x_{2}, x^{\prime}\right)$, from Eq. (6), is

$$
\begin{aligned}
h_{3}\left(x_{2}, x^{\prime}\right)= & \exp \left[j 2 \pi\left(\frac{d_{1}}{\lambda_{s}}+\frac{d_{2}}{\lambda_{i}}\right)\right] \\
& \times \exp \left[j \frac{\pi x_{2}^{2}}{\lambda_{i} d_{2}}\left(1-\frac{1}{M}\right)\right] \delta\left(x_{2}-M x^{\prime}\right) .
\end{aligned}
$$

This is the impulse-response function of an imaging system of magnification $M=-d_{2} \lambda_{i} / d_{1} \lambda_{s}$, and Eq. (17) is the corresponding imaging equation. Note the similarities between Eqs. (15) and (17), despite the fact that the lens is in the signal beam for the former and in the pump beam for the latter.

In the degenerate case where the signal and idler frequencies are equal, $\lambda_{s}=\lambda_{i}=2 \lambda_{p}$, this imaging equation [Eq. (17)] becomes

$$
\frac{1}{d_{1}}+\frac{1}{d_{2}}=\frac{2}{R}
$$

This is the imaging equation of a spherical mirror of radius of curvature $R$, or is the geometrical-optics imaging equation of a thin lens of focal length $R / 2$. Both $I_{0}^{(2)}\left(x_{2}\right)$ and $I^{(2)}\left(x_{2}\right)$ are proportional to the magnified object intensity transmittance $\left|t\left(x_{2} / M\right)\right|^{2}$ if $h_{1}$ is uniform.

\section{B. Object in Both Signal and Idler Beams}

If the signal and idler systems are identical and the object $t$ is placed at the same location in each, as shown in Fig. 5, then we may substitute $h_{s}\left(x_{1}, x\right)=h_{i}\left(x_{1}, x\right)$ $=\int \mathrm{d} x^{\prime} h_{1}\left(x_{1}, x^{\prime}\right) t\left(x^{\prime}\right) h_{2}\left(x^{\prime}, x\right)$ in Eq. (2) to obtain 


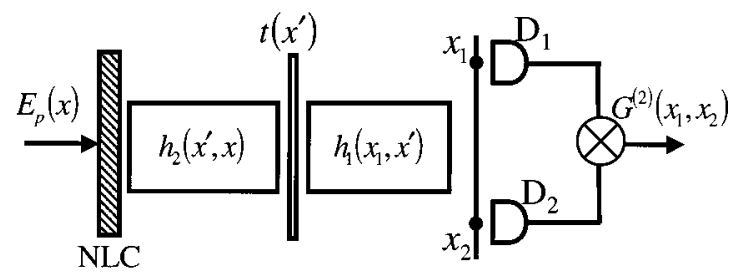

Fig. 5. Object in both signal- and idler-beam configuration. $h_{1}\left(x_{1}, x^{\prime}\right), h_{2}\left(x^{\prime}, x\right)$ are the impulse-response functions of the optical systems placed in the path of both the signal and idler beams, and $t\left(x^{\prime}\right)$ is the object to be imaged.

$$
\begin{aligned}
\psi\left(x_{1}, x_{2}\right)= & \iint \mathrm{d} x^{\prime} \mathrm{d} x^{\prime \prime} t\left(x^{\prime}\right) t\left(x^{\prime \prime}\right) \psi_{c}\left(x^{\prime}, x^{\prime \prime}\right) \\
& \times h_{1}\left(x_{1}, x^{\prime}\right) h_{1}\left(x_{2}, x^{\prime \prime}\right),
\end{aligned}
$$

where

$$
\psi_{c}\left(x^{\prime}, x^{\prime \prime}\right)=\int \mathrm{d} x E_{p}(x) h_{2}\left(x^{\prime}, x\right) h_{2}\left(x^{\prime \prime}, x\right) .
$$

Comparing Eq. (20) with Eq. (A1) shows that $\psi\left(x_{1}, x_{2}\right)$ is analogous to a partially coherent imaging system, where $\psi_{c}\left(x^{\prime}, x^{\prime \prime}\right)$ plays the role of the correlation function of the field. In this case, though, in accordance with Eq. (1) the biphoton rate $G^{(2)}\left(x_{1}, x_{2}\right)$ is a fourth-order nonlinear transformation of $t$.

This system has been used ${ }^{42}$ to test the complementarity of coherence and entanglement with the change of transverse size of the pump beam, where $t$ was taken to be a double slit. From Eqs. (20) and (21) it is clear that for a small source the biphoton amplitude factorizes into a function of $x_{1}$ and another function of $x_{2}$ (coherence), whereas it is not factorizable (i.e., entangled) for a large pump-beam size (entanglement). ${ }^{33}$

\section{Object in the Pump Beam}

In another imaging configuration, the object is placed in the pump beam as illustrated in Fig. 6. Equations (1) and (2) give

$$
G^{(2)}\left(x_{1}, x_{2}\right)=\left|\int \mathrm{d} x t(x) h_{s}\left(x_{1}, x\right) h_{i}\left(x_{2}, x\right)\right|^{2},
$$

provided that $E_{p}(x)$ is uniform over the object. Many possibilities for imaging based on Eq. (22) can be envisioned. For example, if both $h_{s}$ and $h_{i}$ are $2-f$ systems, the result is proportional to the squared magnitude of the Fourier transform of $t$. In another example, if $h_{s}(0, x)$ $=1$, then

$$
I_{0}^{(2)}\left(x_{2}\right)=\left|\int \mathrm{d} x t(x) h_{i}\left(x_{2}, x\right)\right|^{2},
$$

and the behavior is that of a coherent imaging system. The object is illuminated at the pump wavelength, while the measurement is made at the much longer signal and idler wavelengths.

In a third example in which the signal and idler systems are identical, and the coincidence is measured at the same position, by use of a detector sensitive to the arrival of photon pairs (a two-photon absorber, for example), then

$$
G^{(2)}\left(x_{1}, x_{1}\right)=\left|\int \mathrm{d} x t(x) h_{s}^{2}\left(x_{1}, x\right)\right|^{2} .
$$

Again, the mathematical structure is that of a coherent imaging system.

An interesting modification to this configuration would be to add a $2-f$ system between the object and the crystal. In this case, $E_{p}(x)=T\left[2 \pi\left(x / \lambda_{p} f_{\mathrm{o}}\right)\right]$, where $f_{\mathrm{o}}$ is the focal length of the lens before the crystal. If the object is not symmetric in $x$, then its Fourier transform is a complex function. Yet the phase distribution of the object's spatial spectrum is not lost, since the three-wave interaction process in the NLC is coherent. If, in addition, we take both the signal and idler configurations to be $2-f$ systems, the biphoton rate becomes

$$
G^{(2)}\left(x_{1}, x_{2}\right)=\left|t\left[\left(\frac{x_{1}}{\lambda_{s} f_{s}}+\frac{x_{2}}{\lambda_{i} f_{i}}\right) f_{0} \lambda_{p}\right]\right|^{2},
$$

where $f_{s}$ and $f_{i}$ are the focal lengths of the $2-f$ systems in the signal and idler beams, respectively. In this case, in accordance with Eq. (4), $I_{0}^{(2)}$ provides a magnified image of the object $t$ with a magnification factor of $\lambda_{i} f_{i} / \lambda_{p} f_{0}$.

\section{Object is the Detector}

In yet another imaging modality, illustrated in Fig. 7, the object is a two-photon absorber; it is thus a detector with quantum efficiency proportional to its absorbance $t_{2}$. The biphoton rate, in this case $G^{(2)}\left(x_{1}, x_{1}\right)$, is registered by some response of the object, such as emitted photoelectrons or fluorescence. ${ }^{43}$ The signal/idler optical system may, for example, be a single-lens imaging system or a scanning system, as in scanning confocal microscopy. ${ }^{44}$ From Eqs. (1) and (2),

$$
G^{(2)}\left(x_{1}, x_{1}\right)=t_{2}\left(x_{1}\right) S\left(x_{1}\right),
$$

where $S\left(x_{1}\right)$ is an object illumination function. One choice for $S$ would be a very narrow function, which would serve to sample the object in the transverse plane. This could be achieved, for example, by taking a pump of large transverse width and $2-f$ signal and idler systems. The size of the pump and the aperture of the lens limit the transverse resolution.

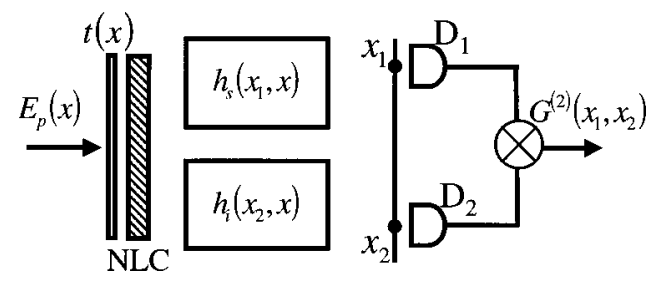

Fig. 6. Object in the pump-beam configuration. $h_{s}\left(x_{1}, x\right)$, $h_{i}\left(x_{2}, x\right)$ are as in Fig. 1, and $t(x)$ is the object to be imaged placed in the pump beam.

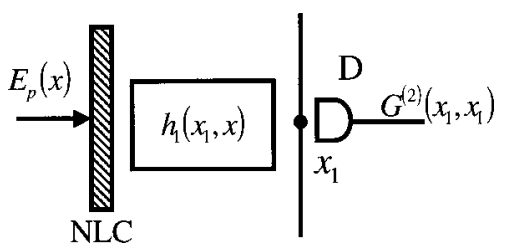

Fig. 7. Object is the detector configuration. $h_{1}\left(x_{1}, x\right)$ is the impulse response of the optical system placed in the signal and idler paths, and $\mathrm{D}$ is a two-photon detector at location $x_{1}$. 


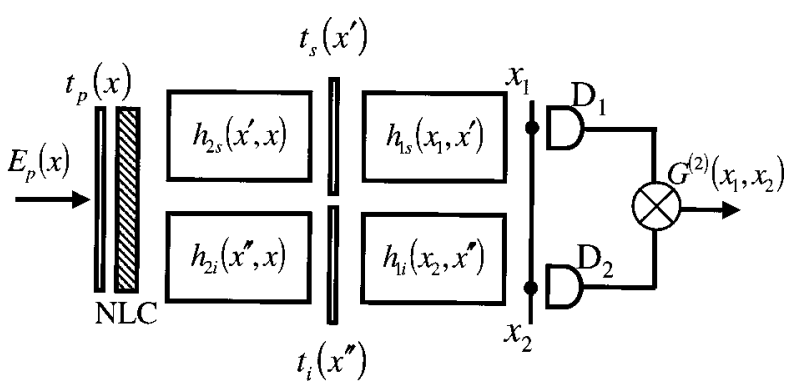

Fig. 8. Configuration for triple correlation. $h_{2 s}\left(x^{\prime}, x\right)$ and $h_{1 s}\left(x_{1}, x^{\prime}\right)$ are the impulse-response functions of the optical systems placed in the signal beam; $h_{2 i}\left(x^{\prime \prime}, x\right)$ and $h_{1 i}\left(x_{2}, x^{\prime \prime}\right)$ are the impulse-response functions of the optical systems placed in the idler beam; $t_{p}(x), t_{s}\left(x^{\prime}\right)$, and $t_{i}\left(x^{\prime \prime}\right)$ are the three objects to be correlated.

There are other sensible choices for the illumination function $S$. These can be implemented through either the pump profile or the system impulse-response function, or both. The object $t_{2}$ would then be extracted by dividing the observed biphoton rate by $S$. The object can also be uniformly illuminated by use of a large pump and a 4- $f$ imaging system, in which case $S$ becomes almost constant over a large portion of the object. We have studied this system elsewhere and compared the longitudinal and transverse resolutions to those of other schemes of microscopy that utilize classical light. ${ }^{45}$

\section{E. Objects in Signal, Idler, and Pump Beams: Image Triple Correlation}

Because the biphoton optical system is based on threewave mixing, it inherently depends on three image distributions and therefore offers a number of unique options for optical image processing. For example, if $4-f$ systems with aperture functions $t_{s}$ and $t_{i}$ are placed in the signal and idler beams, respectively, and a third object is placed in the pump beam such that the field at the entrance to the NLC is $t_{p}$, as shown in Fig. 8, then Eqs. (1) and (2) yield

$$
\begin{aligned}
G^{(2)}\left(x_{1}, x_{2}\right)= & \mid \int \mathrm{d} x t_{p}(x) T_{s}\left[\frac{2 \pi}{\lambda_{s} f_{s}}\left(x-x_{1}\right)\right] \\
& \times\left. T_{i}\left[\frac{2 \pi}{\lambda_{i} f_{i}}\left(x-x_{2}\right)\right]\right|^{2},
\end{aligned}
$$

where $T_{s}(q)$ and $T_{i}(q)$ are the Fourier transforms of $t_{s}(x)$ and $t_{i}(x)$; and $f_{s}$ and $f_{i}$ are the focal lengths of the signal and idler $4-f$ systems, respectively. This equation represents the magnitude of the triple correlation of the three functions $t_{p}, T_{s}$, and $T_{i}$. Triple correlation is useful in a number of signal-processing applications. Of course, if one of these three functions is uniform, the operation becomes ordinary correlation.

One application for this configuration could be system identification and coded-aperture imaging. In this application, a linear, shift-invariant optical system is to be identified, i.e., its impulse-response function measured. The system may be placed in one of the two downconverted beams (say the signal), and a set of $N$ known reference systems are placed, one at a time, in the other beam (the idler) as the coincidence rate is measured.
The set of idler systems are also assumed to be linear and shift invariant with impulse-response functions

$$
h_{i}\left(x_{2}, x\right)=h_{n}\left(x-x_{2}\right), n=1,2, \ldots N .
$$

Such systems may be generated by the use of a bank of apertures (filters). Since the unknown system is shift invariant, its impulse-response function is $h_{s}\left(x_{1}, x\right)$ $=h_{s}\left(x_{1}-x\right)$, so that by virtue of Eqs. (1) and (2) the biphoton rate measured at $x_{1}=x_{2}=0$ is given by

$$
C_{n}=G^{(2)}(0,0)=\left|\int \mathrm{d} x h_{s}(-x) h_{n}(x)\right|^{2},
$$

assuming the pump distribution to be uniform.

If $\left\{h_{n}(x)\right\}$ form a complete set of orthonormal functions, then the measured coefficients $\left\{C_{n}\right\}$ are simply the squared magnitudes of the coefficients of an expansion of the unknown function $h_{s}(-x)$ in this basis. Under special conditions, the phases can be retrieved, and the function $h_{s}(x)$ completely reconstructed. ${ }^{46}$

In the special case for which $h_{n}(x)=\delta\left(x+x_{n}\right)$, so that the idler field is sampled at positions $x_{n}$, Eq. (29) yields

$$
C_{n}=\left|h_{s}\left(x_{n}\right)\right|^{2},
$$

in which case the measured coincidence rates provide samples of the magnitude of the impulse response function. A scanning system can therefore be used to determine $\left|h_{s}(x)\right|$.

\section{RESOLUTION OF BIPHOTON IMAGING}

In all of the configurations studied in the previous section we assumed a thin NLC and a narrow biphoton spectral bandwidth. Under these assumptions the imaging resolution of all the configurations is determined by the apertures placed in the system (including those placed in the pump beam). When these apertures are not accounted for, we obtain results reminiscent of classical geometric optics, such as the imaging formulas in Eqs. (15) and (17). These geometric-optics results are typical of the work that has been carried out to date in entangled-photon imaging. ${ }^{31,32}$

One of the advantages of our formalism is to facilitate deriving the analog of wave-optics results for such systems when all the physical parameters of the optical arrangement are accounted for, by use of straightforward calculations similar to those of classical wave optics. 9,34 In this section we examine the effect of the various parameters of entangled-photon imaging systems on the imaging resolution.

We take the width of the image formed by a point object $t(x)=\delta(x)$ in the marginal coincidence rate [Eq. (3)] as a measure of the resolution of the entangled-photon imaging system. Another definition of resolution may be based on the conditional coincidence rate [Eq. (4)].

We begin by modifying the principal imaging equations [Eqs. (1) and (2)] by taking into consideration the thickness of the NLC and the biphoton spectral bandwidth. We assume a monochromatic pump beam of angular frequency $\omega_{p}$ and transverse distribution $E_{p}(x)$ at the entrance to a NLC of thickness $l$. The coincidence rate 
$G^{(2)}\left(x_{1}, t_{1} ; x_{2}, t_{2}\right)$, with the detection times of $\mathrm{D}_{1}$ and $\mathrm{D}_{2}$ now made explicit, is given by

$$
G^{(2)}\left(x_{1}, t_{1} ; x_{2}, t_{2}\right)=\left|\psi\left(x_{1}, t_{1} ; x_{2}, t_{2}\right)\right|^{2} .
$$

Here $\psi\left(x_{1}, t_{1} ; x_{2}, t_{2}\right)$ may be written in terms of a biphoton spectral amplitude $\widetilde{\psi}\left(x_{1}, x_{2} ; \omega_{s}\right)$ through

$$
\begin{aligned}
\psi\left(x_{1}, t_{1} ; x_{2}, t_{2}\right)=\exp \left(-i \omega_{p} t_{1}\right) \\
\quad \times \int_{\Omega} \mathrm{d} \omega_{s} \exp \left[-i \omega_{s}\left(t_{1}-t_{2}\right)\right] \widetilde{\psi}\left(x_{1}, x_{2} ; \omega_{s}\right),
\end{aligned}
$$

where $\Omega$ is the biphoton spectral bandwidth and $\widetilde{\psi}\left(x_{1}, x_{2} ; \omega_{s}\right)$ is given by ${ }^{33}$

$$
\begin{aligned}
\widetilde{\psi}\left(x_{1}, x_{2} ; \omega_{s}\right)= & \iint \mathrm{d} q_{s} \mathrm{~d} q_{i} \Lambda\left(q_{s}, q_{i} ; \omega_{s}\right) \\
& \times H_{s}\left(x_{1}, q_{s} ; \omega_{s}\right) H_{i}\left(x_{2}, q_{i} ; \omega_{p}-\omega_{s}\right),
\end{aligned}
$$

and the dispersion of the optical systems has been made explicit in the signal and idler transfer functions $H_{s}$ and $H_{i}$, which are Fourier transforms of the impulseresponse functions $h_{s}$ and $h_{i}$ (with respect to the second argument), respectively. The quantity $\Lambda\left(q_{s}, q_{i} ; \omega_{s}\right)$ in Eq. (33) is given by

$$
\Lambda\left(q_{s}, q_{i} ; \omega_{s}\right)=\widetilde{E}_{p}\left(q_{s}+q_{i}\right) \widetilde{\xi}\left(q_{s}, q_{i} ; \omega_{s}\right)
$$

here $q$ is proportional to the transverse component of the momentum vector (it is the spatial frequency in the transverse plane), $\widetilde{E}_{p}(q)$ is the Fourier transform of $E_{p}(x)$, and $\widetilde{\xi}\left(q_{s}, q_{i} ; \omega_{s}\right)$ is a phase-matching function given by

$$
\widetilde{\xi}\left(q_{s}, q_{i} ; \omega_{s}\right)=l \operatorname{sinc}\left(\frac{l}{2 \pi} \Delta r\right) \exp \left(-j \frac{l}{2} \Delta r\right) ;
$$

and $\quad \Delta r=r_{p}\left(q_{s}+q_{i}, \omega_{p}\right)-r_{s}\left(q_{s}, \omega_{s}\right)-r_{i}\left(q_{i}, \omega_{p}-\omega_{s}\right)$; $r_{j}(q, \omega)=\left(n_{j}^{2} \omega^{2} / c^{2}-q^{2}\right)^{1 / 2}, j=p, s$, and $i$, where $n_{j}$ is the NLC index of refraction for the polarization and frequency of the $j$ th field.

In most cases the detectors may be considered to be slow (i.e., their response time is large with respect to the inverse of the bandwidth of the system, which is a reasonable assumption for available photodetectors), and thus they measure a coincidence rate that is averaged over a long time interval. The resulting time-averaged coincidence rate is ${ }^{33}$

$$
C\left(x_{1}, x_{2}\right)=\int_{\Omega} \mathrm{d} \omega_{s}\left|\widetilde{\psi}\left(x_{1}, x_{2} ; \omega_{s}\right)\right|^{2},
$$

showing that the time-averaged coincidence rate for a show detector is an incoherent sum of the biphoton spectral amplitudes over the bandwidth of the system. The spectrum of the downconverted biphotons can be quite large, and the dispersion of the optical components must be considered carefully just as dispersion must be in ultrafast pulsed optics.

We also define conditional and marginal time-averaged coincidence rates as

$$
\begin{aligned}
C\left(x_{2}\right) & =\int \mathrm{d} x_{1} C\left(x_{1}, x_{2}\right), \\
C_{0}\left(x_{2}\right) & =C\left(0, x_{2}\right),
\end{aligned}
$$

respectively. It is obvious that when only a narrow spectral bandwidth is considered, $C\left(x_{2}\right)$ and $C_{0}\left(x_{2}\right)$ coincide with $I\left(x_{2}\right)$ and $I_{0}\left(x_{2}\right)$, respectively.

We now proceed to study the resolution of a representative configuration considered in Section 2: object in the signal (or idler) beam. The biphoton spectral amplitude of this system, illustrated in Fig. 2, now taking into consideration the thickness of the crystal and spectral bandwidth of the system, is given by

$\widetilde{\psi}\left(x_{1}, x_{2} ; \omega_{s}\right)=\int \mathrm{d} x^{\prime} t\left(x^{\prime}\right) h_{1}\left(x_{1}, x^{\prime} ; \omega_{s}\right) h_{3}\left(x_{2}, x^{\prime} ; \omega_{s}\right)$,

where

$$
\begin{aligned}
h_{3}\left(x_{2}, x^{\prime} ; \omega_{s}\right)= & \iint \mathrm{d} q_{s} \mathrm{~d} q_{i} \Lambda\left(q_{s}, q_{i} ; \omega_{s}\right) \\
& \times H_{2}\left(x^{\prime}, q_{s} ; \omega_{s}\right) H_{i}\left(x_{2}, q_{i} ; \omega_{p}-\omega_{s}\right) .
\end{aligned}
$$

We assume throughout that the object is thin and nondispersive. To determine the resolution of this imaging configuration we take $t(x)=\delta(x)$, whereupon Eq. (39) becomes $\widetilde{\psi}\left(x_{1}, x_{2} ; \omega_{s}\right)=h_{1}\left(x_{1}, 0 ; \omega_{s}\right) h_{3}\left(x_{2}, 0 ; \omega_{s}\right)$, and consequently

$$
\begin{gathered}
C\left(x_{2}\right)=\int_{\Omega} \mathrm{d} \omega_{s}\left|h_{3}\left(x_{2}, 0 ; \omega_{s}\right)\right|^{2} g\left(\omega_{s}\right), \\
C_{0}\left(x_{2}\right)=\int_{\Omega} \mathrm{d} \omega_{s}\left|h_{3}\left(x_{2}, 0 ; \omega_{s}\right)\right|^{2} g_{0}\left(\omega_{s}\right),
\end{gathered}
$$

where $\quad g(\omega)=\int \mathrm{d} x\left|h_{1}(x, 0 ; \omega)\right|^{2} \quad$ and $\quad g_{0}(\omega)$ $=\left|h_{1}(0,0 ; \omega)\right|^{2}$. Note that the system $h_{1}$ affects the imaging resolution only through introducing an effective spectral bandwidth that may be ignored if it is larger than that of $h_{3}$.

As a concrete example, we consider the system examined in Subsection 2.A.2, which is the second example of object in the signal-beam configuration, namely, ideal single-lens imaging, illustrated in Fig. 3. We assume, at first, a plane-wave pump, so that $h_{3}\left(x_{2}, 0 ; \omega_{s}\right)$ simplifies to

$$
\begin{aligned}
h_{3}\left(x_{2}, 0 ; \omega_{s}\right) & =\int \mathrm{d} q_{s} \widetilde{\xi}\left(q_{s},-q_{s} ; \omega_{s}\right) \\
& \times H_{2}\left(0, q_{s} ; \omega_{s}\right) H_{i}\left(x_{2},-q_{s} ; \omega_{p}-\omega_{s}\right) .
\end{aligned}
$$

In this example, the transfer functions of the systems $h_{2}$ and $h_{i}$ are given by

$H_{2}\left(0, q_{s} ; \omega_{s}\right)=\exp \left(j k_{s} d_{s}\right) \exp \left(-j \frac{d_{s} q_{s}^{2}}{2 k_{s}}\right)$, 


$$
\begin{aligned}
& H_{i}\left(x_{2},-q_{s} ; \omega_{p}-\omega_{s}\right) \\
& =\exp \left[j k_{i}\left(d_{1}+d_{2}\right)\right] \\
& \quad \times \exp \left(j \frac{k_{i} x_{2}^{2}}{2 d_{2}}\right) \exp \left(-j \frac{d_{i} q_{s}^{2}}{2 k_{i}}\right) P_{g}\left(q_{s}+\frac{k_{i} x_{2}}{d_{2}}\right),
\end{aligned}
$$

where $P_{g}(q)$ is the Fourier transform of

$$
p(x) \exp \left[j \frac{k_{i} x^{2}}{2}\left(\frac{1}{d_{2}}-\frac{1}{f}\right)\right]
$$

with respect to $x$, and $p(x)$ is the lens aperture. Substituting Eqs. (35), (44), and (45) into Eq. (43), we obtain $h_{3}\left(x_{2}, 0 ; \omega_{s}\right)$, which we then use in Eqs. (41) and (42) to estimate the resolution.

There are two techniques to implement this system in an actual setup. In one technique the NLC is adjusted for noncollinear SPDC, and one beam (usually chosen by a pinhole) is directed into the system $h_{s}$ and the other into $h_{i}$. Another technique is to adjust the NLC for collinear SPDC and then separate the two photons comprising the biphoton. In type II SPDC (where the signal and idler photons have orthogonal polarizations) one can use a polarizing beam splitter to separate the biphoton. On the other hand, in type I SPDC (where the signal and idler photons have the same polarization) the use of a nonpolarizing beam splitter will separate the pair into the two output ports of the beam splitter in $50 \%$ of the trials, and send the pair together into one output port in the rest of the trials. In the latter case, the trials do not contribute to the coincidence measurements carried out by the detectors $\mathrm{D}_{1}$ and $\mathrm{D}_{2}$ together with the coincidence detection circuit and thus may be ignored.

Assuming a thin NLC, narrow spectral bandwidth, a plane-wave pump, and degenerate collinear downconversion (where the signal and idler photons are separated with the method outlined above), one obtains the familiar diffraction pattern of a diffraction-limited imaging system. For a rectangular lens aperture of width $D$ and focal length $f$ the result is $C\left(x_{2}\right) \propto\left|\operatorname{sinc}\left(x_{2} / 2 \lambda_{0} F_{\#}\right)\right|^{2}$, where $F_{\#}=f / D$ is the $F$-number of the lens and $\lambda_{0}=2 \lambda_{p}$ is the wavelength of the degenerate downconverted photons. This is the best one can obtain; we demonstrate in the following that relaxing any of the restrictions indicated above will degrade the resolution.

Our calculations have been carried out with a $\beta$-barium borate NLC that is illuminated with a pump of wavelength $\lambda_{p}=325 \mathrm{~nm}$ (which corresponds to the ultraviolet line of a $\mathrm{He}-\mathrm{Cd}$ laser), with a cut-angle of $36.44^{\circ}$ that corresponds to degenerate collinear type I SPDC. Increasing the cut angle beyond $36.44^{\circ}$ yields noncollinear degenerate SPDC, and decreasing the cut angle below this value yields collinear nondegenerate SPDC. ${ }^{28}$

We first consider the effect of the finite thickness of the NLC. One effect is that the distance $d_{1}$, used in the imaging formula presented in Eq. (15), is modified to become

$$
d_{1}=d_{i}+d_{s} \frac{\lambda_{s}}{\lambda_{i}}+l_{\mathrm{eq}},
$$

in contrast to that given in Eq. (12). The quantity $l_{\mathrm{eq}}$ is an equivalent length for the NLC that is related to the physical length $l$ by

$$
l_{\mathrm{eq}}=\frac{l}{2 \lambda_{i}}\left(\frac{\lambda_{s}}{n_{s}}+\frac{\lambda_{i}}{n_{i}}\right) .
$$

For the degenerate case $\left(\lambda_{s}=\lambda_{i}=\lambda_{0}\right)$ this expression simplifies to $l_{\text {eq }}=l / n_{0}$, where $n_{0}$ is the index of refraction of the NLC at the degenerate wavelength. In other words, the thickness of the NLC must be accounted for in calculating the distances in the experimental arrangement in order to satisfy the imaging formula of Eq. (15).

Figure 9 shows $C\left(x_{2}\right)$ for several values of $l$, assuming degenerate collinear downconversion and narrow spectral bandwidth. The distances in the configuration are chosen such that $d_{1}=d_{2}=2 f$ [taking into account the effect of $l$ on $d_{1}$ according to Eq. (46)], which corresponds to an imaging system of unity magnification. For $l=0.1 \mathrm{~mm}$ one obtains the diffraction-limited distribution corresponding to the thin NLC case. When $l$ increases, the distribution widens, signifying a loss of imaging resolution, as is evident for the $l=1-\mathrm{mm}$ and $l=10$-mm curves. This result may be easily understood when one considers the fact that the NLC acts as a spatial filter through the phase-matching function $\widetilde{\xi}\left(q_{s}, q_{i} ; \omega_{s}\right)$, defined in Eq. (35). The collinear SPDC case corresponds to a low-pass spatial filter with a cut-off frequency that is inversely proportional to $l$, and hence the resolution degrades as the NLC thickness increases.

The spectral bandwidth of the system has a similar effect on the imaging resolution, which decreases with increased bandwidth. Figure 10 shows $C\left(x_{2}\right)$ for several values of $\rho=\Omega / \omega_{p}$. These plots were obtained for a NLC of thickness $l=1 \mathrm{~mm}$, collinear SPDC, and a planewave pump. According to Eq. (46), $d_{1}$ is a function of wavelength [and so is $l_{\mathrm{eq}}$ through Eq. (47)], so that only one pair of signal/idler wavelengths satisfy the imaging formula in Eq. (15). All biphotons with other signal/idler pair wavelengths are defocused, and hence their contribution to $C\left(x_{2}\right)$ leads to a reduction in resolution. The plots in Fig. 10 were obtained assuming that Eq. (15) is satisfied by the degenerate signal/idler wavelengths and that at these wavelengths $d_{1}=d_{2}=2 f$.

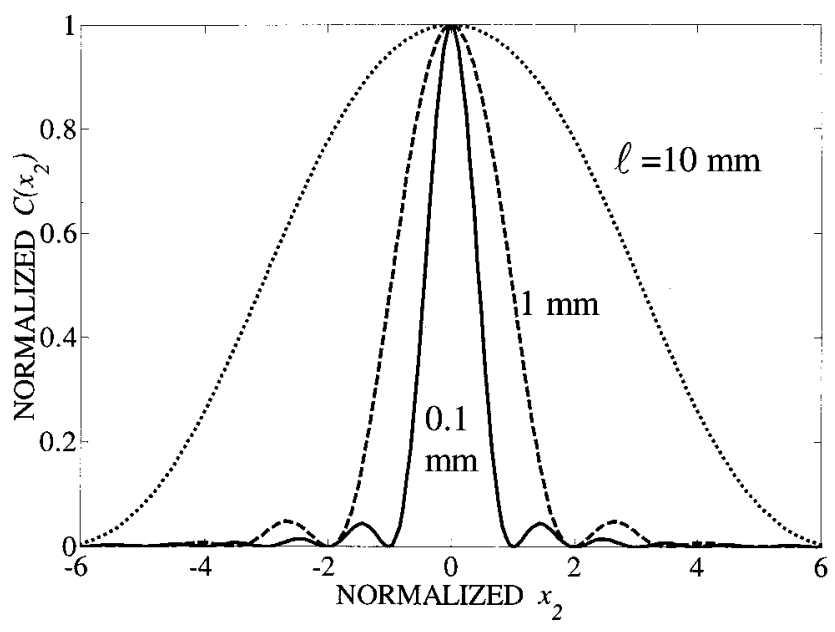

Fig. 9. Effect of NLC thickness $l$ on the imaging resolution of object in the signal-beam configuration. Plots of normalized time-averaged marginal coincidence rate $C\left(x_{2}\right)$ versus detector $\mathrm{D}_{2}$ location $x_{2}$ normalized with respect to $x_{c}=2 \lambda_{0} F_{\#}$, for $l=0.1,1$, and $10 \mathrm{~mm} ; \lambda_{0}=650 \mathrm{~nm}, F_{\#}=5$. 


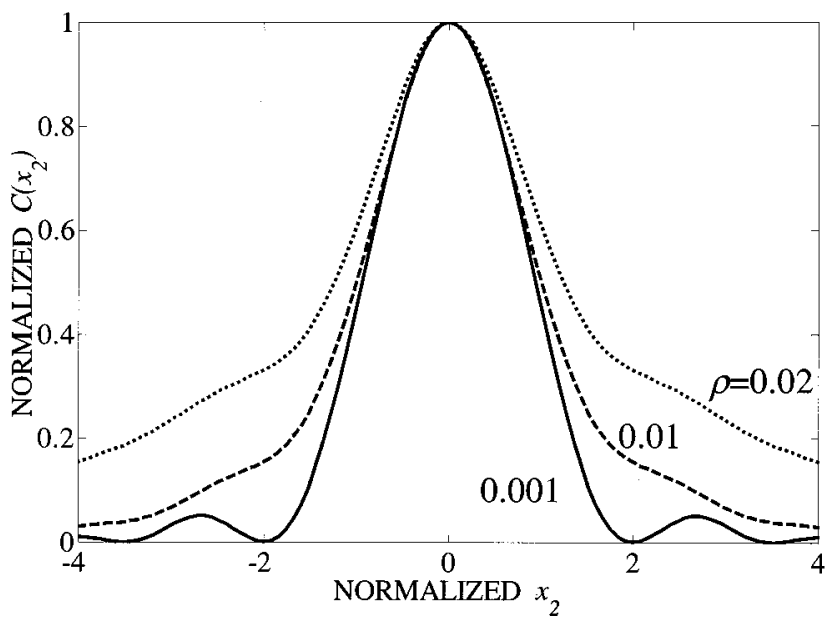

Fig. 10. Effect of the biphoton bandwidth $\Omega$ on the imaging resolution of object in the signal-beam configuration. Plots of normalized $C\left(x_{2}\right)$ versus $x_{2}$ normalized with respect to $x_{c}$ $=2 \lambda_{0} F_{\#}$ are shown for $\rho=\Omega / \omega_{p}=0.001,0.01$, and $0.02 ; \lambda_{0}$ $=650 \mathrm{~nm}, F_{\#}=5$. The NLC is adjusted for collinear SPDC and is of thickness $1 \mathrm{~mm}$.

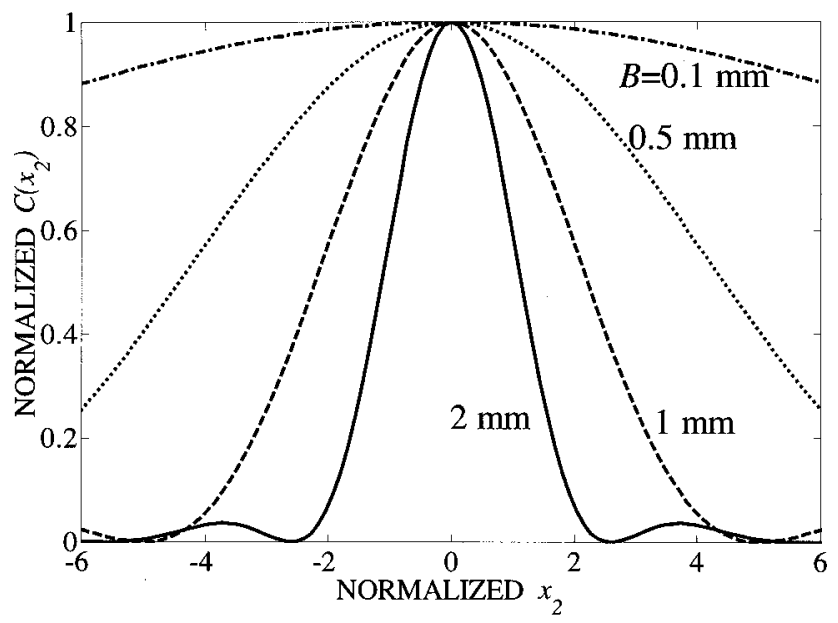

Fig. 11. Effect of the transverse width of the pump $B$ on the imaging resolution of object in the signal-beam configuration. Plots of normalized $C\left(x_{2}\right)$ versus $x_{2}$ normalized with respect to $x_{c}=2 \lambda_{0} F_{\#}$ are shown for $B=2,1,0.5$, and $0.1 \mathrm{~mm}$; $\lambda_{0}=650 \mathrm{~nm}, F_{\#}=5$.

Finally, the finite transverse width of the pump field also degrades the resolution. This can be understood by noting that smaller pump size reduces entanglement of the signal and idler photons. ${ }^{33}$ As a result, the quantum state of the light emitted by the NLC becomes separable, and thus $C\left(x_{2}\right)$ and $C_{0}\left(x_{2}\right)$ simply become the intensity of the idler beam (which depends on $h_{i}$ ) but are independent of the signal beam. ${ }^{38}$ No information about the system $h_{s}$, which includes the object to be imaged in this case, may be extracted from the measurements carried out in the idler beam.

Figure 11 shows plots of $C\left(x_{2}\right)$ for various values of the transverse width of the pump, denoted $B$. The calculations were performed taking $l=1 \mathrm{~mm}$, assuming collinear degenerate SPDC, and the presence of narrow-band spectral filters in the system. Distances were chosen such that $d_{1}=d_{2}=2 f$.

\section{CONCLUSION}

We have presented a Fourier-optics analysis of various imaging configurations using the unique features of spontaneous parametric downconversion (SPDC) as a twophoton source. SPDC is a three-wave mixing process; the pump, signal, and idler are coupled through the phase-matching conditions. We investigated several imaging and image-processing configurations that utilize the quantum correlations among these three fields. Our formalism was also used to study the resolution of these entangled-photon imaging configurations.

\section{APPENDIX A: OPTICAL BILINEAR TRANSFORMATION}

We present a brief overview of the theory of classical imaging in the framework of the bilinear optical transformation. The equations are formulated in such a way so as to facilitate convenient comparisons with the two-photon and biphoton cases presented in the text.

Because of the quadratic relation between the optical field and the optical intensity, imaging systems are typically described by a bilinear transformation. ${ }^{47}$ A general bilinear transformation is expressed as

$$
g\left(x_{1}\right)=\iint \mathrm{d} x^{\prime} \mathrm{d}^{\prime \prime} f^{*}\left(x^{\prime}\right) f\left(x^{\prime \prime}\right) q\left(x_{1} ; x^{\prime}, x^{\prime \prime}\right),
$$

where $f(x)$ is the input function, $q\left(x_{1} ; x^{\prime}, x^{\prime \prime}\right)$ is the double impulse-response function (DIR), and $g(x)$ is the output function. In general, $f(x)$ is complex, but $g(x)$ is guaranteed to be real when the symmetry condition $q\left(x_{1} ; x^{\prime}, x^{\prime \prime}\right)=q^{*}\left(x_{1} ; x^{\prime \prime}, x^{\prime}\right)$ is satisfied. The DIR completely characterizes the bilinear system. This transformation represents, in general, the imaging system depicted in Fig. 12. The input function $f(x)$ represents the transparency $t(x)$; the DIR is a combination of the second-order correlation function of the illumination $G^{(1)}\left(x^{\prime}, x^{\prime \prime}\right)$ and the impulse-response function $h\left(x_{1}, x\right)$ of the linear optical system; and the output $g(x)$ represents the intensity measured by the optical detector.

In the ideal case $q\left(x_{1} ; x^{\prime}, x^{\prime \prime}\right)=\delta\left(x_{1}-x^{\prime}\right) \delta\left(x_{1}\right.$ $\left.-x^{\prime \prime}\right)$, whereupon $g\left(x_{1}\right)=\left|t\left(x_{1}\right)\right|^{2}$, so that the system is a squarer with zero spread. When the DIR factorizes in the form $q\left(x_{1} ; x^{\prime}, x^{\prime \prime}\right)=h^{*}\left(x_{1}, x^{\prime}\right) h\left(x_{1}, x^{\prime \prime}\right)$, the output of the system is given by

$$
g\left(x_{1}\right)=\left|\int \mathrm{d} x^{\prime} f\left(x^{\prime}\right) h\left(x_{1}, x^{\prime}\right)\right|^{2} .
$$

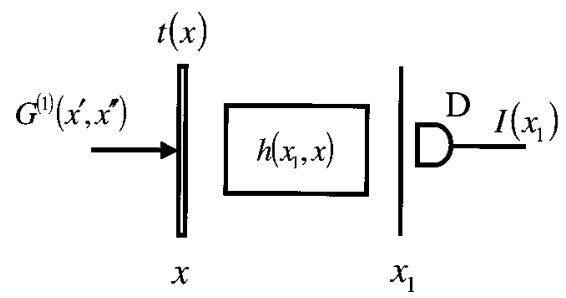

Fig. 12. Classical partially coherent imaging. The quantity $G^{(1)}\left(x^{\prime}, x^{\prime \prime}\right)$ is the second-order correlation function of the optical field; $t$ is the object to be imaged; $h\left(x_{1}, x\right)$ is the impulse response function of the imaging system; $\mathrm{D}$ is a detector placed at position $x_{1}$ that records the intensity $I\left(x_{1}\right)$. 
Equation (A2) is easily recognized as the output intensity of a coherent imaging system with impulse-response function $h\left(x_{1}, x^{\prime}\right)$ and an input complex field $f\left(x^{\prime}\right)$. When the DIR takes the form $q\left(x_{1} ; x^{\prime}, x^{\prime \prime}\right)$ $=h^{*}\left(x_{1}, x^{\prime}\right) h\left(x_{1}, x^{\prime \prime}\right) \delta\left(x^{\prime}-x^{\prime \prime}\right)$, we obtain

$$
g\left(x_{1}\right)=\int \mathrm{d} x^{\prime}\left|f\left(x^{\prime}\right)\right|^{2}\left|h\left(x_{1}, x^{\prime}\right)\right|^{2}
$$

which is the output of an incoherent system with pointspread function $\left|h\left(x_{1}, x^{\prime}\right)\right|^{2}$ and input intensity $\left|f\left(x^{\prime}\right)\right|^{2}$. In general, partially coherent imaging can be represented by a bilinear system with a DIR given by $q\left(x_{1} ; x^{\prime}, x^{\prime \prime}\right)$ $=\gamma\left(x^{\prime}, x^{\prime \prime}\right) h^{*}\left(x_{1}, x^{\prime}\right) h\left(x_{1}, x^{\prime \prime}\right)$, where $\gamma\left(x^{\prime}, x^{\prime \prime}\right)$ represents the correlation function of the input light, and $h\left(x_{1}, x^{\prime}\right)$ is the coherent impulse-response function. When $\gamma\left(x^{\prime}, x^{\prime \prime}\right)=1$, we recover coherent imaging, whereas when $\gamma\left(x^{\prime}, x^{\prime \prime}\right)=\delta\left(x^{\prime}-x^{\prime \prime}\right)$, we recover incoherent imaging.

Entangled-photon imaging, like partially coherent imaging, is described by a bilinear system, with partial entanglement assuming the role of partial coherence. ${ }^{33}$

\section{ACKNOWLEDGMENTS}

This work was supported by the National Science Foundation; by the Center for Subsurface Sensing and Imaging Systems (CenSSIS), an NSF engineering research center; and by the David \& Lucile Packard Foundation.

B. E. A. Saleh's e-mail address is besaleh@bu.edu.

\section{REFERENCES}

1. J. A. Armstrong, N. Bloembergen, J. Ducuing, and P. S. Pershan, "Interactions among light waves in a nonlinear dielectric," Phys. Rev. 127, 1918-1939 (1962).

2. D. A. Kleinman, "Nonlinear dielectric polarization in optical media," Phys. Rev. 126, 1977-1979 (1962)

3. R. H. Kingston, "Parametric amplification and oscillation of optical frequencies," Proc. IRE 50, 472 (1962).

4. S. A. Akhmanov, A. I. Kovrigin, R. V. Khokhlov, and O. N. Chunaev, Zh. Eksp. Teor. Fiz. 45, 1336 (1963).

5. N. Bloembergen, Nonlinear Optics (Benjamin, New York, 1965).

6. F. Zernike and J. E. Midwinter, Applied Nonlinear Optics (Wiley, New York, 1973).

7. A. Yariv, Quantum Electronics, 3rd ed. (Wiley, New York, 1988).

8. P. N. Butcher and D. Cotter, The Elements of Nonlinear Op tics (Cambridge University, Cambridge, England, 1990).

9. B. E. A. Saleh and M. C. Teich, Fundamentals of Photonics (Wiley, New York, 1991).

10. D. L. Mills, Nonlinear Optics: Basic Concepts (Springer, Berlin, 1998).

11. A. Gatti, E. Brambilla, L. A. Lugiato, and M. I. Kolobov, "Quantum entangled images," Phys. Rev. Lett. 83, 17631766 (1999).

12. A. Beranskis, W. Chinaglia, L. A. Lugiato, K.-H. Feller, and P. Di Trapani, "Spatial structures in optical parametric amplification," Phys. Rev. A 60, 1626-1635 (1999).

13. L. A. Lugiato, M. Brambilla, and A. Gatti, "Optical pattern formation," in Advances in Atomic, Molecular, and Optical Physics, B. Bederson and H. Walther, eds. (Academic, Boston, 1999), Vol. 40, pp. 229-306.

14. S.-K. Choi, M. Vasilyev, and P. Kumar, "Noiseless optical amplification of images," Phys. Rev. Lett. 83, 1938-1941 (1999).

15. I. V. Sokolov, M. I. Kolobov, and L. A. Lugiato, "Quantum fluctuations in traveling-wave amplification of optical images," Phys. Rev. A 60, 2420-2430 (1999).

16. D. N. Klyshko, Photons and Nonlinear Optics (Nauka, Moscow, 1980) [translation: Gordon and Breach, New York, 1988].

17. E. Schrödinger, "Die gegenwärtige Situation in der Quantenmechanik," Naturwissenschaften 23, 807-849 (1935) [translation: J. D. Trimmer, "The present situation in quantum mechanics: a translation of Schrodinger's 'cat paradox' paper," Proc. Am. Phil. Soc. 124, 323-338 (1980); reprinted in Quantum Theory and Measurement, J. A. Wheeler and W. H. Zurek, eds. (Princeton University, Princeton, N.J., 1983)].

18. D. N. Klyshko, "Transverse photon bunching and twophoton processes in the field of parametrically scattered light," Zh. Eksp. Teor. Fiz. 83, 1313-1323 (1982) [Sov. Phys. JETP 56, 753-758 (1982)].

19. A. A. Malygin, A. N. Penin, and A. V. Sergienko, "Spatiotemporal grouping of photons in spontaneous parametric scattering of light," Dokl. Akad. Nauk SSSR 281, 308-313 (1985) [Sov. Phys. Dokl. 30, 227-229 (1985)].

20. D. N. Klyshko, "Effect of focusing on photon correlation in parametric light scattering," Zh. Eksp. Teor. Fiz. 94, 82-90 (1988) [Sov. Phys. JETP 67, 1131-1135 (1988)].

21. P. H. S. Ribeiro, S. Pádua, J. C. Machado da Silva, and G. A. Barbosa, "Controlling the degree of visibility of Young's fringes with photon coincidence measurements," Phys. Rev. A 49, 4176-4179 (1994).

22. A. Joobeur, B. E. A. Saleh, and M. C. Teich, "Spatiotemporal coherence properties of entangled light beams generated by parametric down-conversion," Phys. Rev. A 50, 3349-3361 (1994).

23. D. V. Strekalov, A. V. Sergienko, D. N. Klyshko, and Y. H. Shih, "Observation of two-photon "ghost" interference and diffraction," Phys. Rev. Lett. 74, 3600-3603 (1995).

24. A. Joobeur, B. E. A. Saleh, T. S. Larchuk, and M. C. Teich, "Coherence properties of entangled light beams generated by parametric downconversion: theory and experiment," Phys. Rev. A 53, 4360-4371 (1996).

25. A. V. Burlakov, M. V. Chekhova, D. N. Klyshko, S. P. Kulik, A. N. Penin, Y. H. Shih, and D. V. Strekalov, "Interference effects in spontaneous two-photon parametric scattering from two macroscopic regions," Phys. Rev. A 56, 3214-3225 (1997).

26. C. H. Monken, P. H. S. Ribeiro, and S. Pádua, "Transfer of angular spectrum and image formation in spontaneous parametric down-conversion," Phys. Rev. A 57, 3123-3126 (1998).

27. B. M. Jost, A. V. Sergienko, A. F. Abouraddy, B. E. A. Saleh and M. C. Teich, "Spatial correlations of spontaneously down-converted photon pairs detected with a single-photonsensitive CCD camera," Opt. Express 3, 81-88 (1998).

28. A. F. Abouraddy, B. E. A. Saleh, A. V. Sergienko, and M. C. Teich, "Double-slit interference of biphotons generated in spontaneous parametric downconversion from a thick crystal,” J. Opt. B: Quantum Semiclass. Opt. 3, S50-S54 (2001)

29. A. V. Belinskii and D. N. Klyshko, "Two-photon optics: diffraction, holography, and transformation of twodimensional signals," Zh. Eksp. Teor. Fiz. 105, 487-493 (1994) [Sov. Phys. Dokl. 78, 259-262 (1994)]

30. B. E. A. Saleh, S. Popescu, and M. C. Teich, "Generalized entangled-photon imaging," in Proceedings of the Ninth Annual Meeting of the IEEE Lasers and Electro-Optics Society, P. Zory, ed. (IEEE, Piscataway, N.J., 1996), Vol. 1, pp. 362 363.

31. T. B. Pittman, Y. H. Shih, D. V. Strekalov, and A. V. Sergienko, "Optical imaging by means of two-photon quantum entanglement," Phys. Rev. A 52, R3429-R3432 (1995).

32. T. B. Pittman, D. V. Strekalov, D. N. Klyshko, M. H. Rubin, A. V. Sergienko, and Y. H. Shih, "Two-photon geometric optics," Phys. Rev. A 53, 2804-2815 (1996).

33. B. E. A. Saleh, A. F. Abouraddy, A. V. Sergienko, and M. C. 
Teich, "Duality between partial coherence and partial entanglement," Phys. Rev. A 62, 043816 (2000).

34. M. Born and E. Wolf, Principles of Optics, 8th ed. (Cambridge University, New York, 1999).

35. J. W. Goodman, Introduction to Fourier Optics (McGrawHill, New York, 1968).

36. G. O. Reynolds, J. B. DeVelis, G. B. Parrent, Jr., and B. J. Thompson, The New Physical Optics Notebook: Tutorials in Fourier Optics (SPIE, Bellingham, Wash., 1989).

37. F. T. S. Yu and S. Jutamulia, Optical Signal Processing, Computing, and Neural Networks (Wiley, New York, 1992).

38. A. F. Abouraddy, B. E. A. Saleh, A. V. Sergienko, and M. C. Teich, "Role of entanglement in two-photon imaging," Phys. Rev. Lett. 87, 123602 (2001)

39. M. I. Kolobov, "The spatial behavior of nonclassical light," Rev. Mod. Phys. 71, 1539-1589 (1999).

40. M. I. Kolobov and C. Fabre, "Quantum limits on optical resolution,” Phys. Rev. Lett. 85, 3789-3792 (2000).
41. R. J. Glauber, "The quantum theory of optical coherence," Phys. Rev. 130, 2529-2539 (1963).

42. A. F. Abouraddy, M. B. Nasr, B. E. A. Saleh, A. V. Sergienko, and M. C. Teich, "Demonstration of the complementarity of one- and two-photon interference," Phys. Rev. A 63, 063803 (2001).

43. M. C. Teich and B. E. A. Saleh, "Entangled-photon microscopy," Cesk. Cas. Fyz. 47, 3-8 (1997).

44. T. Wilson and C. Sheppard, Theory and Practice of Scanning Optical Microscopy (Academic, London, 1984).

45. M. B. Nasr, A. F. Abouraddy, B. E. A. Saleh, A. V. Sergienko, and M. C. Teich, "Biphoton focusing for two-photon excitation," Phys. Rev. A 65, 023816 (2002).

46. H. Stark, ed., Image Recovery: Theory and Application (Academic, London, 1987)

47. B. E. A. Saleh, "Optical bilinear transformations: general properties,” Opt. Acta 26, 777-799 (1979). 